\title{
Role of apparent diffusion coefficients with diffusion-weighted magnetic resonance imaging in differentiating between benign and malignant bone tumors
}

Tingting Wang ${ }^{1}$, Xiangru Wu ${ }^{2+}$, Yanfen Cui ${ }^{1}$, Caiting Chu ${ }^{1}$, Gang Ren ${ }^{1+}$ and Wenhua $\mathrm{Li}^{1^{*}}$

\begin{abstract}
Background: Benign and malignant bone tumors can present similar imaging features. This study aims to evaluate the significance of apparent diffusion coefficients (ADC) in differentiating between benign and malignant bone tumors.

Methods: A total of 187 patients with 198 bone masses underwent diffusion-weighted (DW) magnetic resonance (MR) imaging. The ADC values in the solid components of the bone masses were assessed. Statistical differences between the mean ADC values in the different tumor types were determined by Student's $t$-test.

Results: Histological analysis showed that 84/198 (42.4\%) of the bone masses were benign and 114/198 (57.6\%) were malignant. There was a significant difference between the mean ADC values in the benign and malignant bone lesions $(P<0.05)$. However, no significant difference was found in the mean ADC value between non-ossifying fibromas, osteofibrous dysplasia, and malignant bone tumors. When an ADC cutoff value $\geq 1.10 \times 10^{-3} \mathrm{~mm}^{2} / \mathrm{s}$ was applied, malignant bone lesions were excluded with a sensitivity of $89.7 \%$, a specificity of $84.5 \%$, a positive predictive value of $82.6 \%$, and a negative predictive value of $95.3 \%$.

Conclusions: The combination of DW imaging with ADC quantification and T2-weighted signal characteristics of the solid components in lesions can facilitate differentiation between benign and malignant bone tumors.
\end{abstract}

Keywords: Apparent diffusion coefficient, Bone tumors, Differentiation, Diffusion-weighted imaging

\section{Background}

Preoperative characterization of benign, malignant, and tumor-like bone lesions is important in order to make informed choices regarding treatment strategies. Although plain film radiography is still considered the first-line imaging modality for assessing the nature and defining the characteristics of primary bone lesions, certain areas of the musculoskeletal system may be difficult to profile in plain films due to overlapping structures [1]. Computerized tomography can provide more detailed information, including focal destruction, periosteal

\footnotetext{
*Correspondence: wenhualimyj@sohu.com

${ }^{\dagger}$ Equal contributors

'Department of Radiology, Xinhua Hospital affiliated to Shanghai Jiao Tong University School of Medicine, 1665 Kong Jiang Road, Shanghai 200092,

China

Full list of author information is available at the end of the article
}

reaction, subtle matrix mineralization, and endosteal scalloping [2]. Magnetic resonance (MR) imaging is considered the most advanced imaging technique and the most sensitive for evaluating changes in bone-marrow and defining the extent of a lesion, particularly when plain films or computerized tomography findings are suboptimal or indeterminate; however, it is not always the most specific. Despite their respective advantages, none of these imaging techniques can reliably differentiate between benign and malignant bone tumors, as many lesions are non-specific and display varying imaging characteristics on T1- and T2-weighted images. MR characterization of bone lesions can be improved by the use of MR diffusion-weighted (DW) imaging, as this is sensitive to changes in the microdiffusion of water into both intracellular and extracellular spaces. The 
advantage of evaluating diffusion is the ability to probe the cellularity of neoplasms. Apparent diffusion coefficients (ADC) are largely proportional to the ratio of extracellular and intracellular components, cell density, intracellular organelles, matrix fibers, and soluble macromolecules. Tumors with different levels of cellularity have different $\mathrm{ADC}$ values corresponding to changes in restricted diffusion [3-6].

The purposes of this study were to clarify the relationship between ADC values in the solid components of bone masses and to evaluate its supplementary use in differentiating between benign and malignant bone tumors.

\section{Methods}

\section{Patient selection}

This study received approval from our institutional (Xinhua Hospital) review board and the requirement to obtain written informed consent was waived. A total of 178 patients who had been diagnosed with bone tumors between January 2005 and March 2014 were enrolled in this study. The selection criteria were as follows: the diagnosis was confirmed by histological biopsy or surgery, MR imaging was performed using a 3.0 T magnet, and both conventional MR imaging with DW imaging and contrasted-enhancement MR imaging were performed. A retrospective evaluation of the $\mathrm{MR}$ imaging data was undertaken.

\section{MR imaging protocol}

All patients underwent MR imaging with a 3.0T MR unit (GE Medical Systems; Milwaukee, WI, USA). Axial noncontrast T1-weighted (TR/TE, 400 to $500 / 10$ to $12 \mathrm{~ms}$ ) and axial T2-weighted (TR/TE, 4,000 to 5,000/100 to $120 \mathrm{~ms}$ ) imaging were performed with chemical shift-selective fat saturation pulse using the following parameters: slice thickness, $5 \mathrm{~mm}$; gap, $1 \mathrm{~mm}$; field of view, 20 to $40 \mathrm{~cm}$; matrix, $256 \times 256$; and excitation, 2. Sagittal T1-weighted and T2weighted (TR/TE, 3,000 to 5,000/100 to $110 \mathrm{~ms}$ ) fast spinecho imaging without chemical shift-selective fat saturation pulse were also performed using the parameters described above. DW-MR imaging was performed in the axial or sagittal plane prior to administration of contrast medium using a single-shot echo-planar imaging sequence (TR/TE effective range, 6,000 to $8,000 / 70$ to $100 \mathrm{~ms}$; slice thickness/ intersection gap, $5 / 1 \mathrm{~mm}$; field of view, 20 to $40 \mathrm{~cm}$; matrix, $128 \times 128$; excitation, 2 . A b-value of 0 and $1,000 \mathrm{~s} / \mathrm{mm}^{2}$ were also applied in three orthogonal directions. Postcontrast-enhanced axial and sagittal T1-weighted imaging were also performed using the parameters described above with the exception of the $5 \mathrm{~mm}$ slice thickness.

\section{$M R$ and $M R-D W$ image analysis}

Conventional MR and DW-MR imaging data were analyzed on an Advantage Windows workstation 4.2 (GE
Healthcare, Milwaukee, WI, USA). Image analysis was carried out by two radiologists in consensus (with 7 and 9 years' experience in musculoskeletal MR imaging, respectively). The signal intensity of the solid components on T2-weighted MR images was defined as intermediate or high, relative to the muscle signal and signal intensity of the solid portions exhibiting enhancement postinjection. The signal intensity of the solid components at $\mathrm{b}=1,000 \mathrm{~s} / \mathrm{mm}^{2}$ on the DW images was defined as intermediate or low relative to that of the muscle.

\section{Data calculation and analysis}

The solid components of the lesions were identified on T2-weighted and post-contrast T1-weighted images, and were matched on ADC maps. The ADC values of the solid components in each tumor were measured on DW images by a radiologist using an Advantage Windows workstation 4.2 and FuncTool software (GE Medical Systems). In order to minimize variability, the largest possible region of interest (ROI) was placed manually in the solid part of the tumor in each image (range: 10 to $80 \mathrm{~mm}^{2}$ ). If the lesion exhibited irregular or heterogeneous solid components, two or three ROIs were drawn within the targeted components and the mean ADC value was calculated for the analyses.

\section{Statistical analysis}

All analyses were performed using SPSS v. 13.0 software for Windows (SPSS; Chicago, IL, USA). Differences in the mean ADC values of the bone tumors between the benign and malignant groups were evaluated using Student's $t$-test. A value $<0.05$ was considered statistically significant. Biopsy or surgical pathology results were used as reference standards for assessment of the bone tumors. Receiver operating characteristic curve analysis was performed to assess the diagnostic performance of the mean $\mathrm{ADC}$ values in characterization of benign and malignant bone tumors.

\section{Results}

\section{Demographics and histopathological characteristics}

The histopathological types of 198 bone masses in the 178 patients are summarized in Table 1 . The study group consisted of 81 males and 97 females, with a mean age of $31.52 \pm 28.31$ years (range: 1 to 92 years). These included 131 patients with solitary bone tumors, 22 patients with 28 Langerhans cell histiocytosis, and 25 patients ( $\mathrm{n}=15$ lung cancer, $\mathrm{n}=4$ breast cancer, $\mathrm{n}=3$ prostate cancer, and $\mathrm{n}=3$ colorectal cancer) with 39 metastatic bone tumors. A total of 84/198 (42.4\%) bone masses were benign and 114/198 (57.6\%) were malignant. The diameters of the lesions in the benign group were 1.5 to $11.2 \mathrm{~cm}$ (median: $3.7 \mathrm{~cm}$ ) and 1.2 to $16 \mathrm{~cm}$ (median, $4.1 \mathrm{~cm}$ ) in the malignant group. 
Table 1 Histological type and apparent diffusion coefficient (ADC) values of 198 bone masses $\left(\mathrm{mean} \pm \mathrm{SD} \times 10^{-3} \mathrm{~mm} / \mathrm{s}\right.$ )

\begin{tabular}{lcrrrr}
\hline Type of bone masses & No. of lesions & High SI on T2WI (\%) & High SI on DWI (\%) & Range of ADC values & Mean ADC value \\
\hline Benign bone lesions & 84 & $54(64.3)$ & $50(59.5)$ & $0.49-1.59$ & $1.17 \pm 0.36$ \\
Non-ossifying fibroma & 17 & $4(23.5)$ & $2(11.8)$ & $0.49-0.99$ & $0.78 \pm 0.17$ \\
Osteofibrous dysplasia & 18 & $3(16.7)$ & $1(5.6)$ & $0.89-1.19$ & $0.97 \pm 0.17$ \\
Chondromyxoid fibroma & 6 & $5(83.3)$ & $4(66.7)$ & $1.18-1.59$ & $1.33 \pm 0.15$ \\
Langerhans cell histiocytosis & 28 & $28(100)$ & $28(100)$ & $0.96-1.55$ & $1.29 \pm 0.18$ \\
Giant cell tumor of bone & 15 & $14(93.3)$ & $15(100)$ & $0.98-1.47$ & $1.21 \pm 0.20$ \\
Malignant bone lesions & 114 & $101(88.6)$ & $105(92.1)$ & $0.58-1.35$ & $0.86 \pm 0.20$ \\
Chordoma & 27 & $21(77.8)$ & $24(88.9)$ & $0.59-1.11$ & $0.80 \pm 0.14$ \\
Ewing sarcoma & 9 & $6(66.7)$ & $7(77.8)$ & $0.70-0.91$ & $0.82 \pm 0.07$ \\
Osteosarcoma & 8 & $6(75.0)$ & $5(62.5)$ & $0.87-1.02$ & $0.97 \pm 0.08$ \\
Chondrosarcoma & 12 & $10(83.0)$ & $11(91.7)$ & $0.69-1.21$ & $0.94 \pm 0.15$ \\
Plasmacytoma & 8 & $8(100)$ & $8(100)$ & $0.68-1.12$ & $0.86 \pm 0.15$ \\
Primary lymphoma & 11 & $11(100)$ & $11(100)$ & $0.68-1.09$ & $0.87 \pm 0.14$ \\
Metastatic bone tumor & 39 & $39(100)$ & $39(100)$ & $0.58-1.12$ & $0.81 \pm 0.14$ \\
\hline
\end{tabular}

Sl, Signal intensity; T2WI, T2-weighted image; DWI, Diffusion-weighted image.

Relationship between DW imaging and bone tumor types The percentage of T2-weighted MR images of the solid components in bone masses giving intermediate or high signal intensities was significantly lower in the benign tumor group compared to the malignant tumor group (64.3\% and $88.6 \%$, respectively; $P<0.01$ ). The 84 benign lesions with homogeneous or heterogeneous low signal intensities included $76.5 \%$ non-ossifying fibromas, $83.3 \%$ osteofibrous dysplasia tumors, $16.7 \%$ chondromyxoid fibromas, and $6.7 \%$ giant cell tumors of bone.

Evaluation of the bone masses by DW imaging at $\mathrm{b}=1,000 \mathrm{~s} / \mathrm{mm}^{2}$, revealed that a significantly higher proportion of malignant lesions exhibited a high signal intensity within the solid components of the bone masses compared to those in benign lesions $(92.1 \%$ and $59.5 \%$, respectively; $P<0.05)$. Further analysis of the images showed that the presence of a solid component with high signal intensity on T2-weighted images and high signal intensity on DW images with low ADC values $\left(<1.10 \times 10^{-3} \mathrm{~mm}^{2} / \mathrm{s}\right.$, at $\mathrm{b}=1,000 \mathrm{~s} / \mathrm{mm}^{2}$ ) could be considered predictive of malignancy (Figure 1). Conversely, the presence of a solid component with high or low signal intensity on T2weighted images and low signal intensity on DW images with high ADC values $\left(\geq 1.10 \times 10^{-3} \mathrm{~mm}^{2} / \mathrm{s}\right.$, at $\mathrm{b}=1,000 \mathrm{~s} /$ $\mathrm{mm}^{2}$ ), or low signal intensity on T2-weighted images and low signal intensity DW images with low ADC values $\left(<1.10 \times 10^{-3} \mathrm{~mm}^{2} / \mathrm{s}\right.$, at $\left.\mathrm{b}=1,000 \mathrm{~s} / \mathrm{mm}^{2}\right)$ could be considered predictive of a benign mass (Figure 2).

\section{ADC analysis}

The mean ADC values of the solid components in the bone masses were determined for each group. There was considerable overlap in the range of values observed within the benign and malignant bone tumors; however, the mean $\mathrm{ADC}$ value for benign tumors $\left(1.17 \pm 0.36 \times 10^{-3} \mathrm{~mm}^{2} / \mathrm{s}\right)$ was significantly higher than that in malignant tumors $\left(0.87 \pm 0.20 \times 10^{-3} \mathrm{~mm}^{2} / \mathrm{s} ; P<0.05\right)$. When the tumor subtypes were compared, no significant difference in the ADC values was found between the non-ossifying fibromas or osteofibrous dysplasia and malignant bone tumors $(P>0.05)$. When an ADC cutoff value $\geq 1.10 \times 10^{-3} \mathrm{~mm}^{2} / \mathrm{s}$ was applied, the benign and malignant bone tumors could be differentiated with a sensitivity of $89.7 \%$, a specificity of $84.5 \%$, a positive predictive value of $82.6 \%$, and a negative predictive value of $95.3 \%$, suggesting that this may be the optimal cutoff value for the ADC.

\section{Discussion}

Our results demonstrated that the presence of a solid component in bone lesions with high signal intensity on DW and T2-weighted images, combined with low ADC values can be used to distinguish between malignant and benign bone lesions. These findings suggested that DW imaging with quantitative analysis of ADCs may improve the diagnostic performance of bone MR imaging, and provide clinically valuable information on the tumor microenvironment.

DW imaging has been extensively investigated for its ability to characterize tissue in various lesions. It has been shown to increase the accuracy in distinguishing benign from malignant masses, and discriminating between metastatic and benign lymphadenopathies. It has also proved successful in the evaluation of cerebral ischemia and intracranial tumors $[1,3,4]$. Malignant tumors with a high nuclear/cytoplasmic ratio, hypercellularity, and a reduced extracellular matrix often have restricted mobility of water 

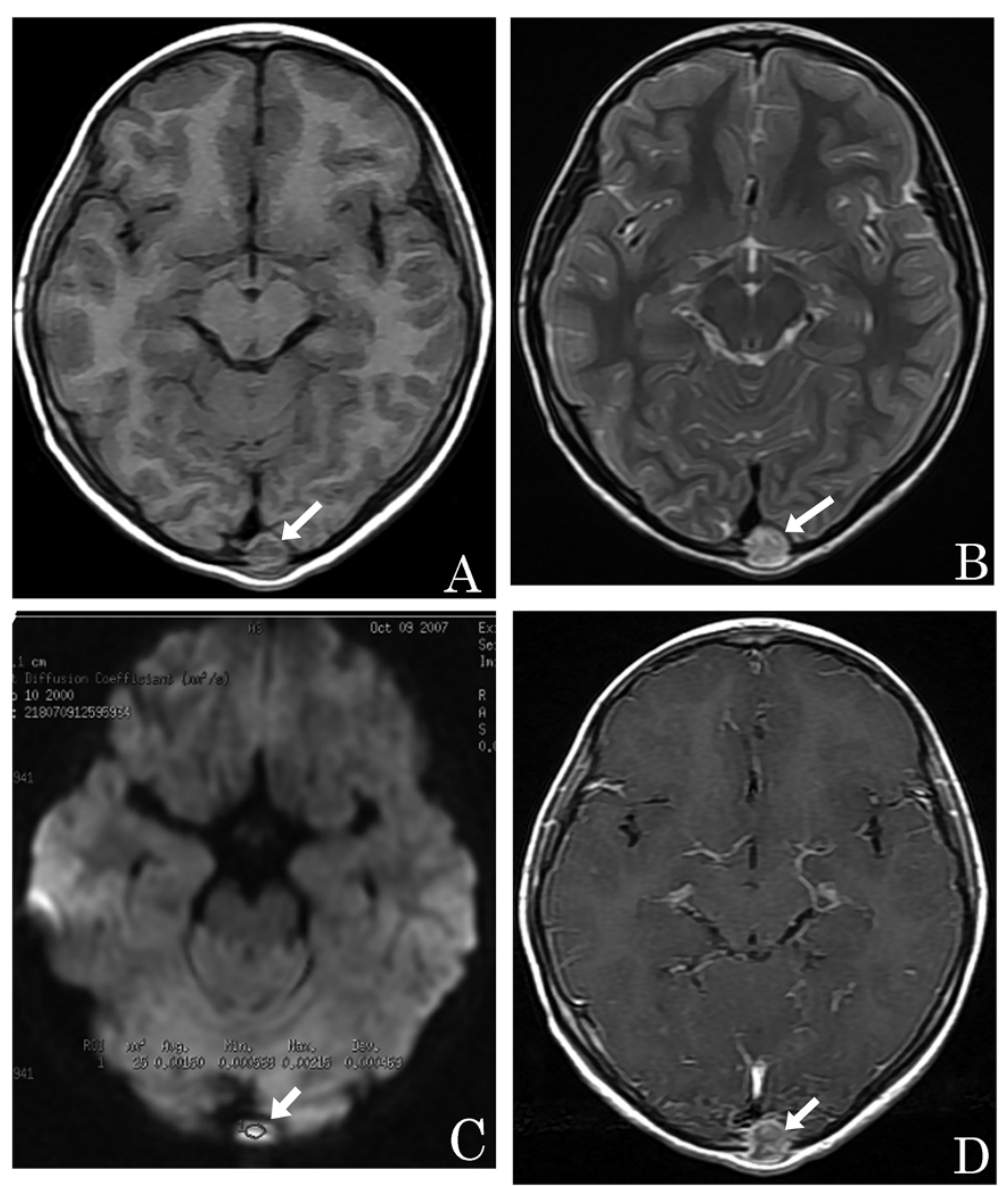

Figure 1 A 7-year-old girl with an eosinophilic granuloma. (A) Axial T1-weighted image showing an isointense small round mass within the occipital bone (arrow). (B) An axial T2-weighted image reveals that the mass is hyperintense (arrow). (C) Axial DW imaging reveals that the mass is slightly hyperintense with a high $A D C$ value (circled area: $A D C=1.50 \times 10^{-3} \mathrm{~mm}^{2} / \mathrm{s}$ ) (arrow). (D) Axial enhanced T1-weighted image showing the lesion with marked enhancement (arrow).

molecules and low ADC values, whereas benign tumors generally have higher $\mathrm{ADC}$ values. These variations were reflected in our results. Although there was some overlap in the ADC values between the malignant and benign bone lesions, the mean ADC values in the 114 malignant bone tumors was significantly lower than that in the 84 benign bone masses. Our results further revealed that the optimal ADC cutoff value for differentiating between benign and malignant tumors was $1.10 \times 10^{-3} \mathrm{~mm}^{2} / \mathrm{s}$. This result was consistent with those given in previous reports [7-12].

A study by Hayashida et al. [4] evaluated the contribution of DW imaging in combination with quantitative analysis of ADCs in the characterization of 20 bone masses, including 8 solitary bone cysts, 5 fibrous dysplasia tumors, and 7 chondrosarcomas. Their results suggested that this method of imaging bone lesions was not suitable for differentiating between benign and malignant bone lesions. This apparent discrepancy may have been due to differences in pathological bone architecture. However, our results revealed a significant difference in the mean $\mathrm{ADC}$ values of the solid components between malignant and benign bone lesions. In addition, our results showed low ADC values in 17 non-ossifying fibromas and 18 osteofibrous dysplasia tumors. These may have been due to the presence of abundant collagen-producing fibroblastic cells and a dense network of collagen fibers within the extracellular matrix, which can restrict the Brownian motion of water molecules. This characteristic may also have been responsible for the absence of a significant difference between the mean ADC values of non-ossifying fibromas or osteofibrous dysplasia tumors and malignant bone lesions.

Our results showed that $88.6 \%$ of the T2-weighted images of solid components in malignant tumors displayed high signal intensities, compared to $11.4 \%$ which displayed low signal intensities. However, this value increased to $92.1 \%$ when the DW images with low ADC 

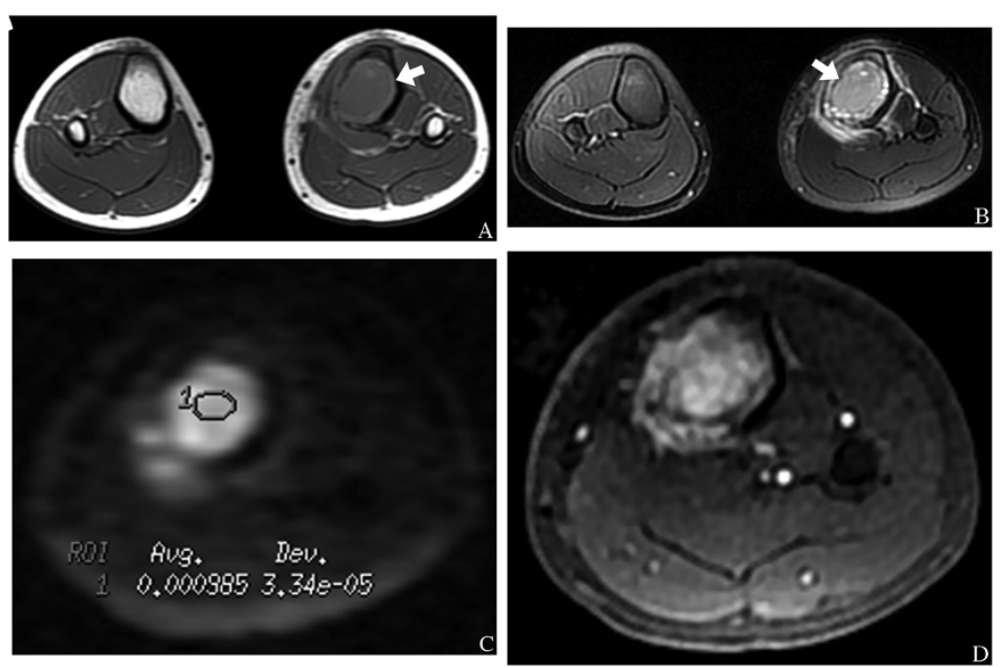

Figure 2 A 23-year-old man with an osteosarcoma. (A) An axial T1-weighted image showing a hypointense left tibial osteosarcoma mass (arrow). (B) Axial T2-weighted image showing that the mass has high signal intensity (arrow). (C) Axial DW imaging reveals that the mass is hyperintense with a low ADC value (circled area: $A D C=0.985 \times 10^{-3} \mathrm{~mm}^{2} / \mathrm{s}$ ). (D) Axial contrast-enhanced T1-weighted image shows marked enhancement of the bone mass.

values were analyzed. Bone lesions which exhibited low signal intensity on T2-weighted images and high signal intensity on DW images may have resulted from solid components with increased nuclear/cytoplasmic ratios and hypercellularity due to a reduction in both the extracellular matrix and the diffusion space of water protons in the extracellular and intracellular dimensions. In addition, the low signal intensity on T2-weighted images and DW images of benign bone lesions, such as nonossifying fibromas and osteofibrous dysplasia tumors, may have been due to the high density of fibers, low cellularity, and low water content in both the extracellular and intracellular spaces $[4,13]$. Our findings confirmed that a high signal intensity on DW images of solid components with low ADC values can serve as a useful criterion for predicting malignancy in bone lesions, and that a low signal intensity on T2-weighted images and DW images of solid components with low ADC values may be an effective criterion for predicting the presence of benign disease.

Our study had the following limitations: DW imaging often has poor spatial resolution, therefore, drawing the ROI on DW images while viewing T2-weighted or contrasted T1-weighted images may result in information bias. A better approach may be to fuse the DW images of solid components that show an abnormal signal at $\mathrm{b}=1,000 \times 10^{-3} \mathrm{~mm}^{2} / \mathrm{s}$ onto structural images in order to accurately position the ROI. Furthermore, the proposed value for the ADC threshold will need to be validated in a larger group of patients, as the ADC can be affected by many factors, including magnetic susceptibility, spatial resolution, signal to noise ratio, and the pathophysiological characteristics of the bone lesions.

\section{Conclusions}

DW imaging is a potentially valuable method for differentiating between benign and malignant bone tumors as it offers both high sensitivity and specificity. Bone masses with solid components that exhibit low signal intensity on T2-weighted and DW images and low ADC values are invariably benign.

\section{Abbreviations}

ADC: Apparent diffusion coefficients; DW: Diffusion-weighted; MR: Magnetic resonance; ROI: Region of interest.

\section{Competing interests}

This research received no specific grant from any funding agency in the public, commercial, or not-for-profit sectors. The authors declare that they have no conflicts of interest.

\section{Authors' contributions}

WH Li planned, designed, and analyzed the study. TT Wang collected data and wrote the manuscript. YF Cui and G Ren collected data. CT Chu designed the study. XR Wu contributed as a pathologist and helped in the writing of the manuscript. All authors read and approved the final manuscript.

\section{Acknowledgements}

We thank Huarong Gong, Huitong AN, Qiufeng Yin, and Ming Liu for their technical support.

\section{Author details}

${ }^{1}$ Department of Radiology, Xinhua Hospital affiliated to Shanghai Jiao Tong University School of Medicine, 1665 Kong Jiang Road, Shanghai 200092,

China. ${ }^{2}$ Department of Pathology, Xinhua Hospital affiliated to Shanghai Jiao Tong University School of Medicine, 1665 Kong Jiang Road, Shanghai 200092, China. 
Received: 23 April 2014 Accepted: 18 November 2014

Published: 29 November 2014

\section{References}

1. Wyers MR: Evaluation of pediatric bone lesions. Pediatr Radiol 2010, 40:468-473.

2. Woertler $\mathrm{K}$ : Benign bone tumors and tumor-like lesions: value of cross-sectional imaging. Eur Radiol 2003, 13:1820-1835.

3. Pearce T, Philip S, Brown J, Koh DM, Burn PR: Bone metastases from prostate, breast and multiple myeloma: differences in lesion conspicuity at short-tau inversion recovery and diffusion-weighted MRI. Br J Radiol 2012, 85:1102-1106.

4. Hayashida Y, Hirai T, Yakushiji T, Katahira K, Shimomura O, Imuta M, Nakaura T, Utsunomiya D, Awai K, Yamashita Y: Evaluation of diffusion-weighted imaging for the differential diagnosis of poorly contrast-enhanced. J Mag Reson Imaging 2006, 23:377-392.

5. Moon WJ, Lee MH, Chung EC: Diffusion-weighted imaging with Sensitivity Encoding (SENSE) for detecting cranial bone marrow metastases: comparison with T1-weighted images. Korean J Radiol 2007, 8:185-191.

6. Jaramilo D: Whole-body MR, imaging, bone diffusion imaging: how and why? Pediatr Radiol 2010, 40:978-984.

7. Ginat DT, Mangla R, Yeaney G, Johnson M, Ekholm S: Diffusion-weighted imaging for differentiating benign from malignant skull lesions and correlation with cell density. Am J Roentgenol 2012, 198(6):597-601.

8. Budde MD, Gold E, Jordan EK, Frank JA: Differential microstructure and physiology of brain and bone metastases in a rat breast cancer model by diffusion and dynamic contrast enhanced MRI. Clin Exp Metastasis 2012, 29(1):51-62.

9. Garrett KM, Kim HK, Stanek J, Emery KH: MR findings of primary bone lymphoma in a 15-year-old girl: emphasis on diffusion-weighted imaging. Pediatr Radiol 2011, 41(5):658-662.

10. Hillengass J, Stieltjes B, Bäuerle T, McClanahan F, Heiss C, Hielscher T, Wagner-Gund B, Habetler V, Goldschmidt H, Schlemmer HP, Delorme S, Zechmann CM: Dynamic contrast-enhanced magnetic resonance imaging (DCE-MRI) and diffusion-weighted imaging of bone marrow in healthy individuals. Acta Radiol 2011, 52(3):324-330.

11. Costa FM, Ferreira EC, Vianna EM: Diffusion-weighted magnetic resonance imaging for the evaluation of musculoskeletal tumors. Magn Reson Imaging Clin N Am 2011, 19(1):159-180.

12. Dremmen MH, Hofman PA, Hof JR, Stokroos RJ, Postma AA: The diagnostic accuracy of non-echo-planar diffusion-weighted imaging in the detection of residual and/or recurrent cholesteatoma of the temporal bone. AJNR Am J Neuroradiol 2012, 33(3):439-444.

13. Ozgen B, Oguz KK, Cila A: Diffusion MR imaging features of skull base osteomyelitis compared with skull base malignancy. AJNR Am J Neuroradiol 2011, 32(1):179-184.

doi:10.1186/1477-7819-12-365

Cite this article as: Wang et al:: Role of apparent diffusion coefficients with diffusion-weighted magnetic resonance imaging in differentiating between benign and malignant bone tumors. World Journal of Surgical Oncology 2014 12:365.

\section{Submit your next manuscript to BioMed Central and take full advantage of:}

- Convenient online submission

- Thorough peer review

- No space constraints or color figure charges

- Immediate publication on acceptance

- Inclusion in PubMed, CAS, Scopus and Google Scholar

- Research which is freely available for redistribution 